\title{
Flatness-Based Adaptive Neurofuzzy Control of Chaotic Dynamical Systems
}

\author{
G. Rigatos ${ }^{1} \cdot$ P. Siano ${ }^{2}$
}

Received: 24 December 2015 / Revised: 23 August 2016 / Accepted: 24 August 2016 / Published online: 17 September 2016

(C) Springer Science+Business Media Singapore 2016

\begin{abstract}
The paper proposes a solution to the problem of control of nonlinear chaotic dynamical systems, which is based on differential flatness theory and on adaptive fuzzy control. An adaptive fuzzy controller is designed for chaotic dynamical systems, under the constraint that the system's model is unknown. The control algorithm aims at satisfying the $H_{\infty}$ tracking performance criterion, which means that the influence of the modeling errors and the external disturbances on the tracking error is attenuated to an arbitrary desirable level. After transforming the chaotic system's model into a linear form, the resulting control inputs are shown to contain nonlinear elements which depend on the system's parameters. The nonlinear terms which appear in the control inputs are approximated with the use of neurofuzzy networks. It is shown that a suitable learning law can be defined for the aforementioned neuro-fuzzy approximators so as to preserve the closed-loop system stability. With the use of Lyapunov stability analysis it is proven that the proposed adaptive fuzzy control scheme results in $H_{\infty}$ tracking performance. The efficiency of the adaptive fuzzy control method is checked through simulation experiments, using as case study the Lorenz chaotic oscillator.
\end{abstract}

Keywords Chaotic dynamical systems - Chaotic oscillators . Lorenz oscillator - Adaptive fuzzy control - Differential flatness theory

\section{G. Rigatos}

grigat@ieee.org

P. Siano

psiano@unisa.it

1 Unit of Industrial Automation, Industrial Systems Institute, 26504 Rion Patras, Greece

2 Department of Industrial Engineering, University of Salerno, 84084 Fisciano, Italy

\section{Introduction}

Chaotic dynamical systems and chaos-based control has been a topic of research interest in the last years . Chaotic dynamics is met in several applications, for example in electric power systems (e.g., converters), in communication systems (chaotic spread spectrum communication), in electronic circuits and in mechanical vibrating structures as well as in biological systems. The response of chaotic systems is depended on initial conditions. Actually, by changing initial conditions the behavior of chaotic systems varies in a manner that approximates stochasticity. In [1], the design of a feedback controller for Chua's chaotic circuit is analyzed, based on a combination of $H_{\infty}$ control theory, adaptive fuzzy control and Lyapunov's stability method. In [2,3] TakagiSugeno fuzzy modelling is proposed for chaotic systems. A feedback controller is designed based on Lyapunov's stability analysis and the solution of LMIs. In [4] linearization of chaotic system's dynamics is proposed with the use of differential flatness theory while a disturbance observer is designed for estimating and compensating for perturbation effects. Observer-based control of chaotic systems is also proposed in [5]. In [6] a neural network is used for identifying the unknown dynamics of the chaotic system, while based on the estimated model a sliding mode feedback controller is designed. In [7] the chaotic system is modeled with the Takagi-Sugeno fuzzy approach. For this latter representation an observer-based feedback control scheme is implemented. Stability analysis is provided with the Lyapunov method. In [8] an adaptive fuzzy system is used to approximate the unknown system dynamics and next a state feedback controller is designed. Stability analysis is shown with the Lyapunov method. In [9] the paper follows a TakagiSugeno fuzzy modelling approach for the chaotic system of unknown dynamics. Using Lyapunov stability method, adap- 
tation of the parameters of the Takagi-Sugeno fuzzy model is succeeded and a feedback control law is designed. In [10] Takagi-Sugeno fuzzy modelling is proposed for synchronizing control of chaotic systems. Stability proof is based on previous results for fractional order systems. In [11] a proportional gain feedback controller is designed for chaotic systems and stability is shown with the use of the Lyapunov method. In [12], Takagi-Sugeno fuzzy modelling and Lyapunov stability analysis are implemented on a chaotic system. The control input has impulsive form. Finally, in [14] the use of differential flatness theory and of Lie algebra for linearization and control of chaotic oscillators is analyzed.

This paper proposes adaptive fuzzy control for chaotic systems of unknown dynamical model with the use of differential flatness theory. On the one side, adaptive fuzzy control has been proven to be an efficient nonlinear control method [13-20]. On the other side, differential flatness theory stands for a major direction in the design of nonlinear control systems [21-27]. It is known that neurofuzzy approximators can be used in indirect adaptive control schemes where their role is to identify online the unknown system dynamics and to provide the control with this information that is used for generating the control inputs [28-30]. Adaptive fuzzy control based on differential flatness theory extends the class of systems to which indirect adaptive fuzzy control can be applied [31-36]. This is important for the design of controllers, capable of efficiently compensating for modeling uncertainties and external disturbances in nonlinear dynamical systems. Unlike other adaptive fuzzy control schemes which are based on several assumptions about the structure of the nonlinear system as well as about the uncertainty characterizing the system's model, the proposed adaptive fuzzy control scheme based on differential flatness theory offers an exact solution to the design of fuzzy controllers for unknown dynamical systems [37-39]. The only assumption needed for the design of the controller and for succeeding $H_{\infty}$ tracking performance for the control loop is that there exists a solution for a Riccati equation associated with the linearized error dynamics of the differentially flat model. This assumption is quite reasonable for several nonlinear systems, thus providing a systematic approach to the design of reliable controllers for such systems $[30,34]$.

In this paper a new nonlinear adaptive-fuzzy control scheme based on differential flatness theory is proposed for chaotic dynamical systems. The new results come to extend the method presented in [31-36]. By showing that chaotic dynamical systems are differentially flat one it becomes possible to transform them to the linear canonical form. For the latter description of the system's dynamics the design of a state feedback controller becomes easier. After transformation to the linear canonical form, the resulting control input for the chaotic dynamical system is shown to contain nonlinear elements which depend on the system's parameters. If the parameters of the system are unknown, then the nonlinear terms which appear in the control signal can be approximated with the use of neuro-fuzzy networks $[40,41]$. In the paper it is shown that a suitable learning law can be defined for the aforementioned neuro-fuzzy approximators so as to preserve the closed-loop system stability. Lyapunov stability analysis proves also that the proposed flatness-based adaptive fuzzy control scheme results in $H_{\infty}$ tracking performance.

The structure of the paper is as follows: in "Differential Flatness of Chaotic Dynamical Systems" section differential flatness properties for chaotic dynamical systems are shown and the transformation of these dynamical systems to the linear canonical form is explained. In "Flatness-Based Adaptive Fuzzy Control" section adaptive fuzzy control based on differential flatness theory is proposed for nonlinear systems of unknown and time-varying dynamics. In "Lyapunov Stability Analysis" section using Lyapunov stability analysis it is shown that the adaptive fuzzy control based on differential flatness theory satisfies $H_{\infty}$ tracking performance criteria. In "Nonlinear Feedback Control of Chaotic Systems Based on Takagi-Sugeno Fuzzy Modelling" section nonlinear feedback control for chaotic dynamical systems is developed with the use of Takagi-Sugeno fuzzy modelling and the solution of Linear Matrix Inequalities. In "Simulation Tests" section simulation tests are provided and the performance of the control of chaotic systems based on differential flatness theory is compared against control based on Takagi-Sugeno fuzzy modelling. Finally, in "Conclusions" section concluding remarks are stated.

\section{Differential Flatness of Chaotic Dynamical Systems}

\section{The Lorenz chaotic system:}

The Lorenz system is obtained from studying a fluid layer heated from below and cooled from above, such that a temperature difference is established across it. The convection motion is described by the Navier-Stokes equations. Taking Fourier expansions of these equations along two spatial directions and truncating the remaining expressions after the third mode, the following simplified model is obtained:

$$
\begin{aligned}
& \dot{x}=\sigma(y-z) \\
& \dot{y}=\rho x-y-x z \\
& \dot{z}=-\beta z+x y
\end{aligned}
$$

where $\sigma, \rho$ and $\beta$ are real parameters denoting the Prandtl number, the Rayleigh number and a geometric factor, respectively. The state variables $x, y$ and $z$ represent measures of fluid velocities and the spatial temperature distribution in the fluid layer under gravity. The Rayleigh number can be manip- 


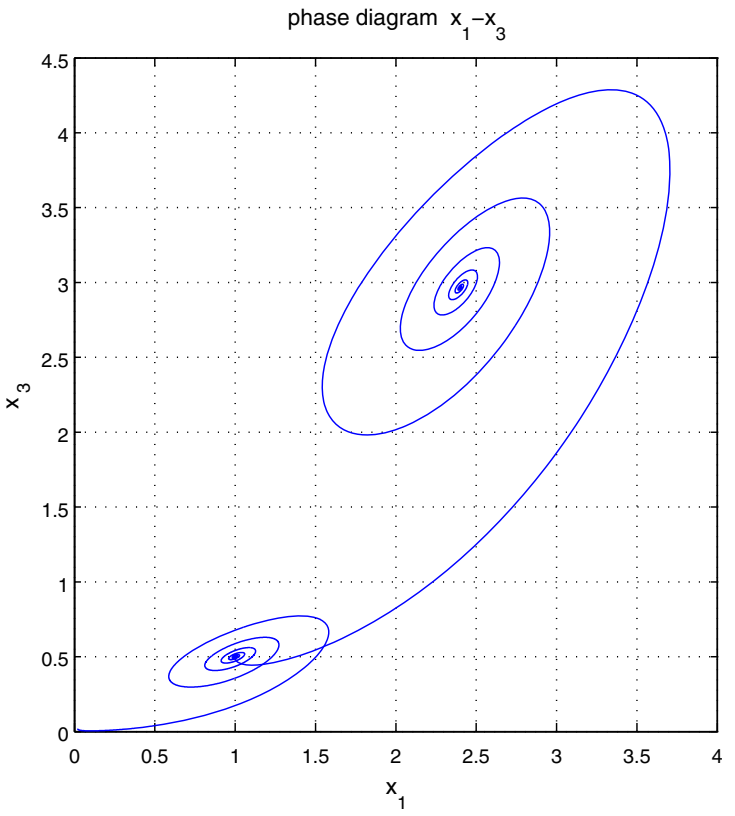

(a)

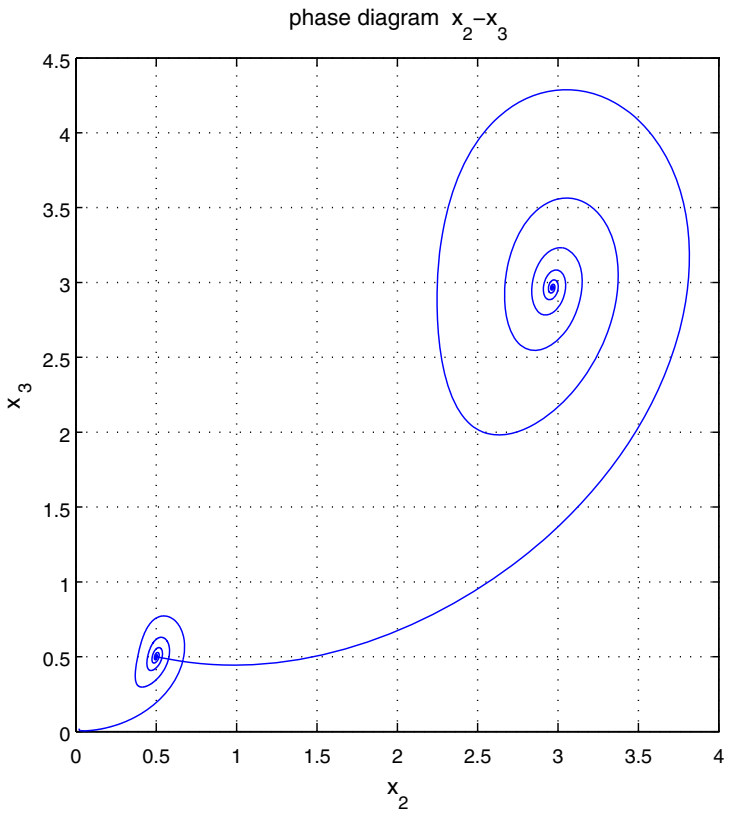

(b)

Fig. 1 a Indicative phase diagram for the Lorenz system for states $x_{1}$ and $x_{3}$, b Indicative phase diagram for the Lorenz system for states $x_{2}$ and $x_{3}$

ulated by changing the heat transfer to the fluid from below. This parameter is considered to be a control input $u=\rho$. Common values for parameters $\sigma$ and $\beta$ are $\sigma=10$ and $\beta=8 / 3$. Indicative phase diagrams of the Lorenz chaotic oscillator are shown in Fig. 1

The flat output of the Lorenz system given in Eq. (1) is defined as [14]:

$\lambda=\frac{x^{2}}{2}-\sigma z+k$

The transformed state vector is

$$
\begin{aligned}
z= & {\left[z_{1}, z_{2}, z_{3}\right]^{T}=\Phi(x) \Rightarrow } \\
z= & {\left[x^{2} / 2-\sigma z+k, \sigma\left(\beta \zeta-x^{2}\right),\right.} \\
& \left.\sigma \gamma x y+2 \sigma^{2} x^{2}-\sigma \beta^{2} \zeta\right]^{T}
\end{aligned}
$$

where $\gamma=\beta-2 \sigma$. The inverse transformation is given by

$$
\begin{aligned}
& x=[x, y, z]^{T}=\Phi^{-1}(z) \\
& x= \pm \sqrt{\frac{2\left(\beta \epsilon+z_{2}\right)}{\gamma}} \\
& y= \pm \frac{2 \sigma \beta z_{1}+(\beta+2 \sigma) z_{2}+z_{3}-2 \sigma \beta k}{\sigma \sqrt{2 \gamma\left(\beta \epsilon+z_{2}\right)}} \\
& z=\frac{2 \sigma \epsilon+z_{2}}{\sigma \gamma}
\end{aligned}
$$

where $\epsilon=z_{1}-k$. The transformed dynamical system can be written in the linear canonical (Brunovsky) form according to $\dot{z}_{3}=\lambda^{(3)}=v$

where

$v=a(x)+\beta(x) u$

where $u=\rho$ and

$$
\begin{aligned}
a(x)= & -\sigma \gamma x^{2} z-4 \sigma^{3} x^{2}-\sigma\left(\gamma+\beta^{2}\right. \\
& \left.+\sigma \beta-6 \sigma^{2}\right) x y+\sigma^{2} \gamma y^{2}+\sigma \beta^{3} x \\
\beta(x)= & \sigma \gamma x^{2}
\end{aligned}
$$

It holds that

$$
\begin{aligned}
& z_{1}=\lambda=x^{2} / 2-\sigma z+k \\
& z_{2}=\dot{\lambda}=\sigma\left(\beta z-x^{2}\right) \\
& z_{3}=\ddot{\lambda}=\sigma \gamma x y+2 \sigma^{2} x^{2}-\beta^{2} \sigma x
\end{aligned}
$$

Finally, it is shown that all state variable of the Lorenz system can be written as functions of the flat outputs and of its derivatives. Indeed, it holds

$$
\begin{aligned}
x= & \pm \sqrt{2 \kappa / \gamma} \\
y= & \pm(\ddot{\lambda}+(\beta+2 \sigma) \dot{\lambda}+2 \sigma \beta \lambda-2 \sigma k) /(\sigma \sqrt{2 \gamma \kappa}) \\
z= & (\beta \dot{\lambda}+2 \sigma \beta \lambda-2 \sigma \kappa) /(\sigma \beta \gamma) \\
u= & \{-2 \beta \gamma \kappa v-2 \beta \gamma(\beta \kappa+\kappa+2 c \sigma-\sigma k) \ddot{\lambda} \\
& -2 \beta \dot{\lambda}\left(4 k c-2 c^{2}-2 k^{2}+2 \sigma \gamma \kappa+\beta \gamma \kappa\right.
\end{aligned}
$$




$$
\begin{aligned}
& +2 \sigma \beta \gamma c-\gamma \sigma \beta \kappa)-\} \\
& -4 \sigma \beta\left(\beta \gamma \kappa+4 \kappa c-2 c^{2}-2 k^{2}\right) \lambda-8 \sigma k c^{2}+4 \sigma \beta \gamma k \kappa \\
& -8 \sigma k^{3}+16 \sigma k^{2} c+\beta^{2} \gamma(\beta+2 \sigma) \dot{\lambda}^{2}+2 \beta \gamma(\beta+\sigma) \ddot{\lambda} \dot{\lambda} \\
& \left.+\beta \gamma \ddot{\lambda}^{2}+2 \sigma \beta^{2} \gamma \ddot{\lambda} \lambda+2 \sigma \beta^{3} \gamma \dot{\lambda} \lambda\right\} /\left(4 \sigma \beta \gamma \kappa^{2}\right)
\end{aligned}
$$

where $c=\dot{\lambda}+\beta \lambda, \kappa=c-k$ and $v=\lambda^{(3)}$. After expressing the dynamics of the Lorenz system with the new state variables $z_{1}, z_{2}, z_{3}$ one has the following linear canonical form

$$
\left(\begin{array}{l}
\dot{z}_{1} \\
\dot{z}_{2} \\
\dot{z}_{3}
\end{array}\right)=\left(\begin{array}{lll}
0 & 1 & 0 \\
0 & 0 & 1 \\
0 & 0 & 0
\end{array}\right)\left(\begin{array}{l}
z_{1} \\
z_{2} \\
z_{3}
\end{array}\right)+\left(\begin{array}{l}
0 \\
0 \\
1
\end{array}\right) v
$$

2. Duffing's chaotic system:

$$
\begin{aligned}
& \dot{x}_{1}(t)=x_{2}(t) \\
& \dot{x}_{2}(t)=1.1 x_{1}(t)-x_{1}^{3}(t)-0.4 x_{2}(t)+1.8 \cos (1.8 t)
\end{aligned}
$$

Differential flatness of Duffing's oscillator:

By defining the flat output $y=x_{1}$ one has $x_{1}=y, x_{2}=\dot{y}$ and $u=1.8 \cos (1.8 t)=\ddot{y}-1.1 y+y^{3}+0.4 \dot{y}$. Thus all state variables and the control input of the Duffing oscillator are expressed as functions of the flat output and its derivatives, and differential flatness of the oscillator is proven. Moreover, by defining the new control input $v=1.1 x_{1}-x_{1}^{3}-0.4 x_{2}+u$, and the new state variables $y_{1}=y$ and $y_{2}=\dot{y}$ a description of the oscillator's dynamics in the linear canonical (Brunovsky's) form is obtained

$$
\left(\begin{array}{l}
\dot{y}_{1} \\
\dot{y}_{2}
\end{array}\right)=\left(\begin{array}{ll}
0 & 1 \\
0 & 0
\end{array}\right)\left(\begin{array}{l}
y_{1} \\
y_{2}
\end{array}\right)+\left(\begin{array}{l}
0 \\
1
\end{array}\right) v
$$

3. The Genesio-Tesi chaotic system:

$$
\begin{aligned}
& \dot{x}_{1}(t)=x_{2}(t) \\
& \dot{x}_{2}(t)=x_{3}(t) \\
& \dot{x}_{3}(t)=-c x_{1}(t)-b x_{2}(t)-\alpha x_{3}(t)+x_{1}^{2}(t)
\end{aligned}
$$

where $a, b$ and $c$ are real constants. When at least one of the system's Lyapunov exponents is larger than zero the system is considered to be chaotic. For example, when $a=1.2, b=$ 2.92 and $c=6$ the system behaves chaotically.

By defining the flat output $y=x_{1}$ one has $x_{1}=y, x_{2}=$ $\dot{y}, x_{3}=\ddot{y}$ and $y^{(3)}=\dot{x}_{3}=-c y-b \dot{y}-a \ddot{y}+y^{2}$. Thus all state variables of the Genesio-Tesi oscillator are expressed as functions of the flat output and its derivatives, and differential flatness of the oscillator is proven. Moreover, by defining as new control input $v=-c y-b \dot{y}-a \ddot{y}+y^{2}$, and the new state variables $y_{1}=y, y_{2}=\dot{y}$ and $y_{3}=\ddot{y}$ a description of the oscillator's dynamics in the linear canonical (Brunovsky's) form is obtained

$$
\left(\begin{array}{l}
\dot{y}_{1} \\
\dot{y}_{2} \\
\dot{y}_{3}
\end{array}\right)=\left(\begin{array}{lll}
0 & 1 & 0 \\
0 & 0 & 1 \\
0 & 0 & 0
\end{array}\right)\left(\begin{array}{l}
y_{1} \\
y_{2} \\
y_{3}
\end{array}\right)+\left(\begin{array}{l}
0 \\
0 \\
1
\end{array}\right) v
$$

4. The Chen chaotic system:

$\dot{x}_{1}(t)=-a\left(x_{1}(t)-x_{2}(t)\right)$

$\dot{x}_{2}(t)=(c-a) x_{1}(t)+c x_{2}(t)-x_{1}(t) x_{3}(t)$

$\dot{x}_{3}(t)=x_{1}(t) x_{2}(t)-b x_{3}(t)$

where $a, b$ and $c$ are positive parameters. When at least one of the system's Lyapunov exponents is larger than zero the system is considered to be chaotic. For example, when $a=$ $40, b=3$ and $c=3$ the system behaves chaotically.

By defining the flat output $y=x_{1}$ one has $x_{1}=y, x_{2}=$ $\frac{1}{a}(\dot{y}+a y)$ and $x_{3}=-\frac{\ddot{y}+(a-c) \dot{y}-\left(2 a c-a^{2}\right) y}{a y}($ for $y \neq 0)$. From the third row of the associated state-space equations and after intermediate computations one obtains $y^{(3)}=-(a-c+$ $\left.1+a^{2} b\right) \ddot{y}-\left[-2\left(2 a c-a^{2}\right)+a^{2} b(a+c)\right] \dot{y}-\frac{(a+c)}{y} \dot{y}^{2}-$ $a y^{2}(\dot{y}+a y)-a^{2} b\left(2 a c-a^{2}\right) y$. Thus all state variables of the Chen oscillator are expressed as functions of the flat output and its derivatives, and differential flatness of the oscillator is proven. Moreover, by defining as new control input $v=$ $-\left(a-c+1+a^{2} b\right) \ddot{y}-\left[-2\left(2 a c-a^{2}\right)+a^{2} b(a+c)\right] \dot{y}-$ $\frac{(a+c)}{y} \dot{y}^{2}-a y^{2}(\dot{y}+a y)-a^{2} b\left(2 a c-a^{2}\right) y$, and the new state variables $y_{1}=y, y_{2}=\dot{y}$ and $y_{3}=\ddot{y}$ a description of the oscillator's dynamics in the linear canonical (Brunovsky's) form is obtained

$$
\left(\begin{array}{l}
\dot{y}_{1} \\
\dot{y}_{2} \\
\dot{y}_{3}
\end{array}\right)=\left(\begin{array}{lll}
0 & 1 & 0 \\
0 & 0 & 1 \\
0 & 0 & 0
\end{array}\right)\left(\begin{array}{l}
y_{1} \\
y_{2} \\
y_{3}
\end{array}\right)+\left(\begin{array}{l}
0 \\
0 \\
1
\end{array}\right) v
$$

It is noted that differential flatness can be proven for several other types of chaotic oscillators, such as Rössler's system and Chua's circuit. By expressing all state variables and the control input of such systems as functions of the flat output and their derivatives it is possible to obtain again a description in the linear canonical (Brunovsky) form. The evolution in time of the phase diagram of the Duffing oscillator and of the Genesio-Tesi chaotic system are shown in Fig. 2.

\section{Flatness-Based Adaptive Fuzzy Control}

\subsection{Nonlinear System Transformation into the Brunovsky Form}

A single-input differentially flat dynamical system is considered again:

$\dot{x}=f_{s}(x, t)+g_{s}(x, t)(u+\tilde{d}), x \in R^{n}, u \in R, \tilde{d} \in R$

where $f_{s}(x, t), g_{s}(x, t)$ are nonlinear vector fields defining the system's dynamics, $u$ denotes the control input and $\tilde{d}$ 


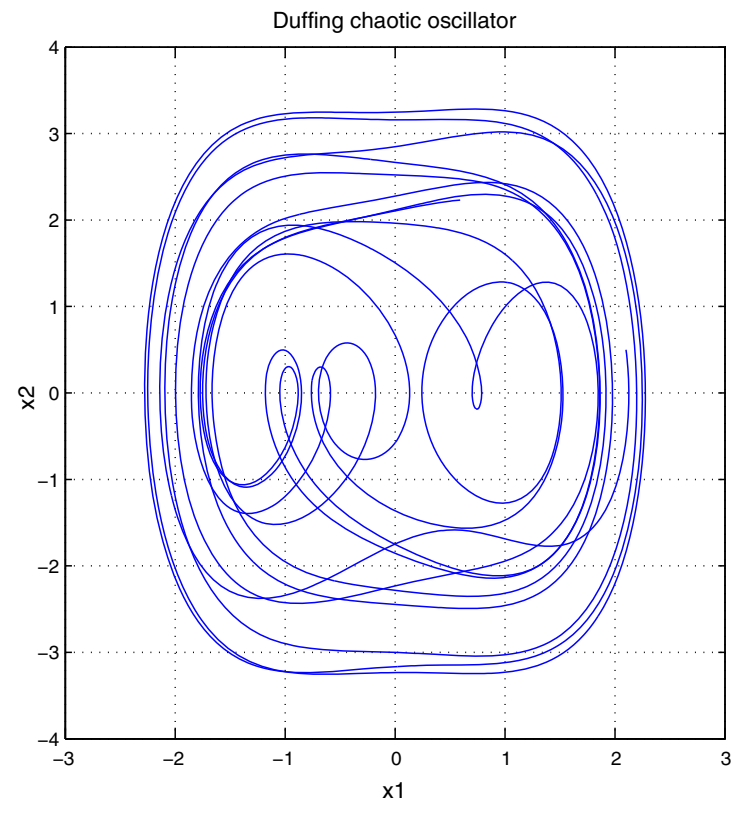

(a)

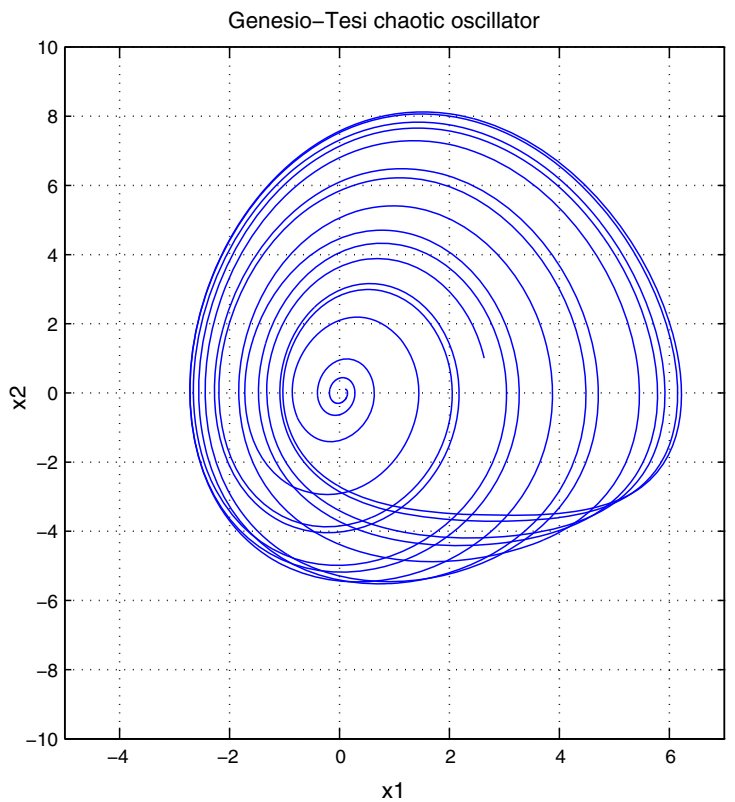

(b)

Fig. 2 Phase diagram of typical chaotic oscillators a Duffing's oscillator, b Genesio-Tesi's oscillator

denotes additive input disturbances. Knowing that the system of Eq. (19) is differentially flat, the next step is to try to write it into a Brunovsky form. It has been shown that, in general, transformation into the Brunovsky (canonical) form can be succeeded for systems that admit static feedback linearization [24]. Single input differentially flat systems, admit static feedback linearization, therefore they can be transformed into the Brunovksy form. For multi-input differentially flat systems there exists also a transformation into the Brunovsky form [30].

The selected flat output is again denoted by $y$. For the state variables $x_{i}$ of the system of Eq. (19) it holds

$x_{i}=\phi_{i}\left(y, \dot{y}, \cdots, y^{(r-1)}\right), i=1, \cdots, n$

while for the control input it holds

$u=\psi\left(y, \dot{y}, \cdots, y^{(r-1)}, y^{(r)}\right)$

Introducing the new state variables $y_{1}=y$ and $y_{i}=$ $y^{(i-1)}, i=2, \cdots, n$, the initial system of Eq. (19) can be written in the Brunovsky form:

$$
\left[\begin{array}{c}
\dot{y}_{1} \\
\dot{y}_{2} \\
\cdots \\
\cdots \\
\dot{y}_{n-1} \\
\dot{y}_{n}
\end{array}\right]=\left[\begin{array}{ccccc}
0 & 1 & 0 & \ldots & 0 \\
0 & 0 & 1 & \ldots & 0 \\
\ldots & \ldots & \ldots & \ldots & \ldots \\
\ldots & \ldots & \ldots & \ldots & \ldots \\
0 & 0 & 0 & \ldots & 1 \\
0 & 0 & 0 & \ldots & 0
\end{array}\right]\left(\begin{array}{c}
y_{1} \\
y_{2} \\
\cdots \\
\ldots \\
y_{n-1} \\
y_{n}
\end{array}\right)+\left(\begin{array}{c}
0 \\
0 \\
\ldots \\
\ldots \\
0 \\
1
\end{array}\right) v
$$

where $v=f(x, t)+g(x, t)(u+\tilde{d})$ is the control input for the linearized model, and $\tilde{d}$ denotes additive input disturbances. Thus one can use that

$y^{(n)}=f(x, t)+g(x, t)(u+\tilde{d})$

where $f(x, t), g(x, t)$ are unknown nonlinear functions, while as mentioned above $\tilde{d}$ is an unknown additive disturbance. Eq. (23) is in the same form as the Lorenz chaotic oscillator's dynamics given in Eq. (5). It is possible to make the system's state vector $x$ follow a given bounded reference trajectory $x_{d}$. In the presence of model uncertainties and external disturbances, denoted by $w_{d}$, successful tracking of the reference trajectory is provided by the $H_{\infty}$ criterion $[20,35]$ :

$\int_{0}^{T} e^{T} Q e d t \leq \rho^{2} \int_{0}^{T} w_{d}^{T} w_{d} d t$

where $\rho$ is the attenuation level and corresponds to the maximum singular value of the transfer function $G(s)$ of the linearized model associated to Eqs. (22) and (23).

Remark 1 From the $H_{\infty}$ control theory, the $H_{\infty}$ norm of a linear system with transfer function $G(s)$, is denoted by $\|G\|_{\infty}$ and is defined as $\|G\|_{\infty}=\sup _{\omega} \sigma_{\max }[G(j \omega)][42-$ 44]. In this definition sup denotes the supremum or least upper bound of the function $\sigma_{\max }\left[G(j(\omega)]\right.$, and thus the $H_{\infty}$ norm of $G(s)$ is the maximum value of $\sigma_{\max }[G(j(\omega)]$ over all frequencies $\omega . H_{\infty}$ norm has a physically meaningful interpretation when considering the system $y(s)=G(s) u(s)$. 
When this system is driven with a unit sinusoidal input at a specific frequency, $\sigma_{\max }|G(j \omega)|$ is the largest possible output for the corresponding sinusoidal input. Thus, the $H_{\infty}$ norm is the largest possible amplification over all frequencies of a sinusoidal input.

Remark 2 The input additive disturbance term $\tilde{d}$ in the dynamics of the controlled system does not affect the transformation into the canonical form that is performed according to the differential flatness theory. Such a disturbance is efficiently compensated by the proposed adaptive fuzzy control law.

\subsection{Control Law}

For the measurable state vector $x$ of the system of Eqs. (22) and (23), as well as for the description of the Lorenz oscillator's dynamics given in Eq. (5), and for uncertain functions $f(x, t)$ and $g(x, t)$ an appropriate control law is

$u=\frac{1}{\hat{g}(x, t)}\left[y_{d}^{(n)}-\hat{f}(x, t)-K^{T} e+u_{c}\right]$

with $e=\left[e, \dot{e}, \ddot{e}, \cdots, e^{(n-1)}\right]^{T}$ and $e=y-y_{d}, K^{T}=$ $\left[k_{n}, k_{n-1}, \cdots, k_{1}\right]$, such that the polynomial $e^{(n)}+k_{1} e^{(n-1)}+$ $k_{2} e^{(n-2)}+\cdots+k_{n} e$ is Hurwitz. The control law of Eq. (25) results into

$$
\begin{aligned}
e^{(n)}= & -K^{T} e+u_{c}+[f(x, t)-\hat{f}(x, t)] \\
& +[g(x, t)-\hat{g}(x, t)] u+g(x, t) \tilde{d}
\end{aligned}
$$

where the supervisory control term $u_{c}$ aims at the compensation of the approximation error

$w=[f(x, t)-\hat{f}(x, t)]+[g(x, t)-\hat{g}(x, t)] u$

as well as of the additive disturbance term $d_{1}=g(x, t) \tilde{d}$. The above relation can be written in a state-equation form. The state vector is taken to be $e^{T}=\left[e, \dot{e}, \cdots, e^{(n-1)}\right]$, which after some operations yields

$$
\begin{aligned}
\dot{e}= & \left(A-B K^{T}\right) e+B u_{c}+B\{[f(x, t)-\hat{f}(x, t)] \\
& \left.+[g(x, t)-\hat{g}(x, t)] u+d_{1}\right\}
\end{aligned}
$$

where

$$
A=\left(\begin{array}{cccccc}
0 & 1 & 0 & \ldots & \ldots & 0 \\
0 & 0 & 1 & \ldots & \ldots & 0 \\
\ldots & \ldots & \ldots & \ldots & \ldots & \ldots \\
\ldots & \ldots & \ldots & \ldots & \ldots & \ldots \\
0 & 0 & 0 & \ldots & \ldots & 1 \\
0 & 0 & 0 & \ldots & \ldots & 0
\end{array}\right) \quad B=\left(\begin{array}{c}
0 \\
0 \\
\ldots \\
\ldots \\
0 \\
1
\end{array}\right)
$$

and $K=\left[k_{n}, k_{n-1}, \cdots, k_{2}, k_{1}\right]^{T}$. As explained above, the control signal $u_{c}$ is an auxiliary control term, used for the compensation of $\tilde{d}$ and $w$, which can be selected according to $H_{\infty}$ control theory:

$u_{c}=-\frac{1}{r} B^{T} P e$

\subsection{Approximators of Unknown System Dynamics}

The approximation of functions $f(x, t)$ and $g(x, t)$ of Eq. (23) can be carried out with neuro-fuzzy networks (Fig. 3). To this end, the weights of the neural networks are trained with the use of gradient-type algorithms $[40,41]$. The estimation of $f(x, t)$ and $g(x, t)$ can be written as [13]:

$\hat{f}\left(x \mid \theta_{f}\right)=\theta_{f}^{T} \phi(x), \quad \hat{g}\left(x \mid \theta_{g}\right)=\theta_{g}^{T} \phi(x)$,

where $\phi(x)$ are kernel functions with elements

$\phi^{l}(x)=\frac{\prod_{i=1}^{n} \mu_{A_{i}}^{l}\left(x_{i}\right)}{\sum_{l=1}^{N} \prod_{i=1}^{n} \mu_{A_{i}}^{l}\left(x_{i}\right)} l=1,2, \cdots, N$

It is assumed that the weights $\theta_{f}$ and $\theta_{g}$ vary in the bounded areas $M_{\theta_{f}}$ and $M_{\theta_{g}}$ which are defined as

$$
\begin{array}{r}
M_{\theta_{f}}=\left\{\theta_{f} \in R^{h}:\left\|\theta_{f}\right\| \leq m_{\theta_{f}}\right\}, \\
M_{\theta_{g}}=\left\{\theta_{g} \in R^{h}:\left\|\theta_{g}\right\| \leq m_{\theta_{g}}\right\}
\end{array}
$$

with $m_{\theta_{f}}$ and $m_{\theta_{g}}$ positive constants.

The values of $\theta_{f}$ and $\theta_{g}$ that give optimal approximation are:

$\theta_{f}^{*}=\arg \min _{\theta_{f} \in M_{\theta_{f}}}\left[s u p_{x \in U_{x}}\left|f(x)-\hat{f}\left(x \mid \theta_{f}\right)\right|\right]$

$\theta_{g}^{*}=\arg \min _{\theta_{g} \in M_{\theta g}}\left[\sup _{x \in U_{x}}\left|g(x)-\hat{g}\left(x \mid \theta_{g}\right)\right|\right]$

The approximation error of $f(x, t)$ and $g(x, t)$ is given by

$$
\begin{aligned}
w= & {\left[\hat{f}\left(x, \mid \theta_{f}^{*}\right)-f(x, t)\right]+\left[\hat{g}\left(x \mid \theta_{f}^{*}\right)-g(x, t)\right] u \Rightarrow } \\
w= & \left\{\left[\hat{f}\left(x \mid \theta_{f}^{*}\right)-\hat{f}\left(x \mid \theta_{f}\right)\right]+\left[\hat{f}\left(x \mid \theta_{f}\right)-f(x, t)\right]\right\} \\
& +\left\{\left[\hat{g}\left(x \mid \theta_{g}^{*}\right)-\hat{g}\left(x \mid \theta_{g}\right)\right]+\left[\hat{g}\left(x \mid \theta_{g}\right)-g(x, t)\right] u\right\}
\end{aligned}
$$

where: (i) $\hat{f}\left(x \mid \theta_{f}^{*}\right)$ is the approximation of $f$ for the best estimation $\theta_{f}^{*}$ of the weights' vector $\theta_{f}$, (ii) $\hat{g}\left(x \mid \theta_{g}^{*}\right)$ is the approximation of $g$ for the best estimation $\theta_{g}^{*}$ of the weights' vector $\theta_{g}$. 
Fig. 3 Neuro-fuzzy approximator: $G_{i}$ Gaussian basis function, $N_{i}$ normalization unit

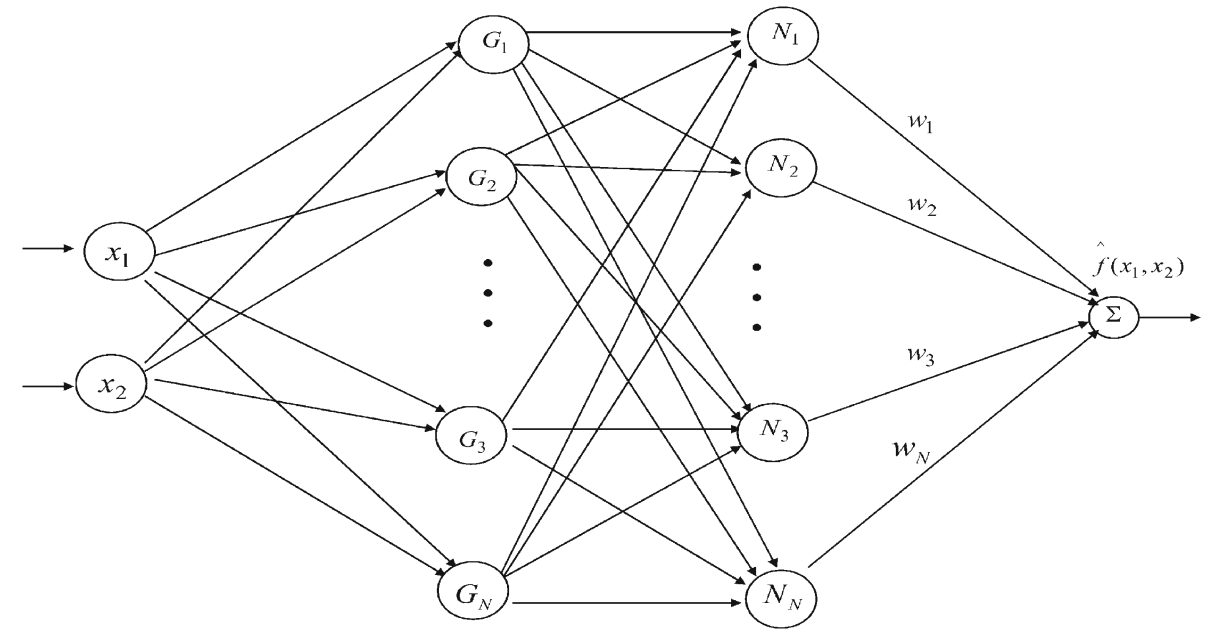

The approximation error $w$ can be decomposed into $w_{a}$ and $w_{b}$, where

$$
\begin{aligned}
& w_{a}=\left[\hat{f}\left(x \mid \theta_{f}\right)-\hat{f}\left(x \mid \theta_{f}^{*}\right)\right]+\left[\hat{g}\left(x \mid \theta_{g}\right)-\hat{g}\left(x \mid \theta_{g}^{*}\right)\right] u \\
& w_{b}=\left[\hat{f}\left(x \mid \theta_{f}^{*}\right)-f(x, t)\right]+\left[\hat{g}\left(x \mid \theta_{g}^{*}\right)-g(x, t)\right] u
\end{aligned}
$$

Finally, the following two parameters are defined:

$$
\begin{aligned}
& \tilde{\theta}_{f}=\theta_{f}-\theta_{f}^{*} \\
& \tilde{\theta}_{g}=\theta_{g}-\theta_{g}^{*} .
\end{aligned}
$$

Remark 3 A difference between the neurofuzzy approximator depicted in Fig. 3 and a RBF neural network is the normalization layer that appears between the Gaussian basis functions layer and the weights output layer. After normalization, the sum (over the complete set of rules) of the fuzzy membership values of each input pattern becomes equal to 1 . The neurofuzzy model provides also linguistic interpretability (in terms of fuzzy rules) about the implemented control law [40].

\section{Lyapunov Stability Analysis}

The adaptation law of the weights $\theta_{f}$ and $\theta_{g}$ as well as of the supervisory control term $u_{c}$ is derived by the requirement for negative definite derivative of the quadratic Lyapunov function

$V=\frac{1}{2} e^{T} P e+\frac{1}{2 \gamma_{1}} \tilde{\theta}_{f}^{T} \tilde{\theta}_{f}+\frac{1}{2 \gamma_{2}} \tilde{\theta}_{g}^{T} \tilde{\theta}_{g}$

Substituting Eq. (28) into Eq. (38) and differentiating gives

$\dot{V}=\frac{1}{2} \dot{e}^{T} P e+\frac{1}{2} e^{T} P \dot{e}+\frac{1}{\gamma_{1}} \tilde{\theta}_{f}^{T} \dot{\tilde{\theta}}_{f}+\frac{1}{\gamma_{2}} \tilde{\theta}_{g}^{T} \dot{\tilde{\theta}}_{g} \Rightarrow$

$$
\begin{aligned}
\dot{V}= & \frac{1}{2} e^{T}\left\{\left(A-B K^{T}\right)^{T} P+P\left(A-B K^{T}\right)\right\} e \\
& +B^{T} P e\left(u_{c}+w+d_{1}\right)+\frac{1}{\gamma_{1}} \tilde{\theta}_{f}^{T} \dot{\tilde{\theta}}_{f}+\frac{1}{\gamma_{2}} \tilde{\theta}_{g}^{T} \dot{\tilde{\theta}}_{g}
\end{aligned}
$$

Assumption 1 For given positive definite matrix $Q$ and coefficients $r$ and $\rho$ there exists a positive definite matrix $P$, which is the solution of the following matrix equation

$$
\begin{aligned}
& \left(A-B K^{T}\right)^{T} P+P\left(A-B K^{T}\right) \\
& -P B\left(\frac{2}{r}-\frac{1}{\rho^{2}}\right) B^{T} P+Q=0
\end{aligned}
$$

Substituting Eq. (40) into $\dot{V}$ yields after some operations

$$
\begin{aligned}
\dot{V}= & -\frac{1}{2} e^{T} Q e+\frac{1}{2} e^{T} P B\left(\frac{2}{r}-\frac{1}{\rho^{2}}\right) B^{T} P e \\
& +B^{T} P e\left(-\frac{1}{r} e^{T} P B\right)+B^{T} P e\left(w+d_{1}\right) \\
& +\frac{1}{\gamma_{1}} \tilde{\theta}_{f}^{T} \dot{\tilde{\theta}}_{f}+\frac{1}{\gamma_{2}} \tilde{\theta}_{g}^{T} \dot{\tilde{\theta}}_{g}
\end{aligned}
$$

It holds that

$\dot{\tilde{\theta}}_{f}=\dot{\theta}_{f}-\dot{\theta}_{f}^{*}=\dot{\theta}_{f}$

$\dot{\tilde{\theta}}_{g}=\dot{\theta}_{g}-\dot{\theta}_{g}^{*}=\dot{\theta}_{g}$

The following weight adaptation laws are considered [31]

$\dot{\theta}_{f}=\left\{\begin{array}{c}-\gamma_{1} e^{T} P B \phi(x) \text { if }\left\|\theta_{f}\right\|<m_{\theta_{f}} \\ 0\left\|\theta_{f}\right\| \geq m_{\theta_{f}}\end{array}\right.$
$\dot{\theta}_{g}=\left\{\begin{array}{c}-\gamma_{2} e^{T} P B \phi(x) u_{c} \text { if }\left\|\theta_{g}\right\|<m_{\theta_{g}} \\ 0\left\|\theta_{g}\right\| \geq m_{\theta_{g}}\end{array}\right.$

$\dot{\theta}_{f}$ and $\dot{\theta}_{g}$ are set to 0 , when

$\left\|\theta_{f}\right\| \geq m_{\theta_{f}}, \quad\left\|\theta_{g}\right\| \geq m_{\theta_{g}}$. 
The update of $\theta_{f}$ stems from a gradient algorithm on the cost function $\frac{1}{2}(f-\hat{f})^{2}$. The update of $\theta_{g}$ is also of the gradient type, while $u_{c}$ implicitly tunes the adaptation gain $\gamma_{2}$. Substituting Eqs. (43) and (44) in $\dot{V}$ finally gives

$$
\begin{aligned}
\dot{V}= & -\frac{1}{2} e^{T} Q e-\frac{1}{2 \rho^{2}} e^{T} P B B^{T} P e+e^{T} P B\left(w+d_{1}\right) \\
& -e^{T} P B\left(\theta_{f}-\theta_{f}^{*}\right)^{T} \phi(x)-e^{T} P B\left(\theta_{g}-\theta_{g}^{*}\right)^{T} \phi(x) u_{c} \Rightarrow \\
\dot{V}= & -\frac{1}{2} e^{T} Q e-\frac{1}{2 \rho^{2}} e^{T} P B B^{T} P e \\
& +e^{T} P B\left(w+d_{1}\right)+e^{T} P B w_{\alpha} .
\end{aligned}
$$

Denoting $w_{1}=w+d_{1}+w_{\alpha}$ one gets

$$
\begin{aligned}
\dot{V}= & -\frac{1}{2} e^{T} Q e-\frac{1}{2 \rho^{2}} e^{T} P B B^{T} P e \\
& +e^{T} P B w_{1} \text { or equivalently, } \\
\dot{V}= & -\frac{1}{2} e^{T} Q e-\frac{1}{2 \rho^{2}} e^{T} P B B^{T} P e \\
& +\frac{1}{2} e^{T} P B w_{1}+\frac{1}{2} w_{1}^{T} B^{T} P e
\end{aligned}
$$

Lemma 1 The following inequality holds:

$$
\begin{aligned}
& \frac{1}{2} e^{T} P B w_{1}+\frac{1}{2} w_{1}^{T} B^{T} P e-\frac{1}{2 \rho^{2}} e^{T} P B B^{T} P e \\
& \quad \leq \frac{1}{2} \rho^{2} w_{1}^{T} w_{1}
\end{aligned}
$$

Proof The binomial $\left(\rho a-\frac{1}{\rho} b\right)^{2} \geq 0$ is considered. Expanding the left part of the above inequality one gets

$$
\begin{aligned}
\rho^{2} a^{2}+\frac{1}{\rho^{2}} b^{2}-2 a b \geq 0 & \Rightarrow \frac{1}{2} \rho^{2} a^{2}+\frac{1}{2 \rho^{2}} b^{2} \\
-a b \geq 0 & \Rightarrow \\
a b-\frac{1}{2 \rho^{2}} b^{2} \leq \frac{1}{2} \rho^{2} a^{2} \Rightarrow & \frac{1}{2} a b+\frac{1}{2} a b \\
& -\frac{1}{2 \rho^{2}} b^{2} \leq \frac{1}{2} \rho^{2} a^{2}
\end{aligned}
$$

The following substitutions are carried out: $a=w_{1}$ and $b=e^{T} P B$ and the previous relation becomes

$$
\begin{aligned}
& \frac{1}{2} w_{1}^{T} B^{T} P e+\frac{1}{2} e^{T} P B w_{1}-\frac{1}{2 \rho^{2}} e^{T} P B B^{T} P e \\
& \quad \leq \frac{1}{2} \rho^{2} w_{1}^{T} w_{1}
\end{aligned}
$$

The previous inequality is used in $\dot{V}$, and the right part of the associated inequality is enforced

$\dot{V} \leq-\frac{1}{2} e^{T} Q e+\frac{1}{2} \rho^{2} w_{1}^{T} w_{1}$
Eq. (51) can be used to show that the $H_{\infty}$ performance criterion is satisfied. The integration of $\dot{V}$ from 0 to $T$ gives

$$
\begin{aligned}
& \int_{0}^{T} \dot{V}(t) d t \leq-\frac{1}{2} \int_{0}^{T}\|e\|_{Q}^{2} d t+\frac{1}{2} \rho^{2} \int_{0}^{T}\left\|w_{1}\right\|^{2} d t \\
& \Rightarrow 2 V(T)+\int_{0}^{T}\|e\|_{Q}^{2} d t \leq 2 V(0)+\rho^{2} \int_{0}^{T}\left\|w_{1}\right\|^{2} d t
\end{aligned}
$$

Moreover, if there exists a positive constant $M_{w}>0$ such that

$\int_{0}^{\infty}\left\|w_{1}\right\|^{2} d t \leq M_{w}$

then one gets

$\int_{0}^{\infty}\|e\|_{Q}^{2} d t \leq 2 V(0)+\rho^{2} M_{w}$

Thus, the integral $\int_{0}^{\infty}\|e\|_{Q}^{2} d t$ is bounded. Moreover, $V(T)$ is bounded and from the definition of the Lyapunov function $V$ in Eq. (38) it becomes clear that $e(t)$ will be also bounded since $e(t) \in \Omega_{e}=\left\{e \mid e^{T} P e \leq 2 V(0)+\rho^{2} M_{w}\right\}$.

According to the above and with the use of Barbalat's Lemma one obtains $\lim _{t \rightarrow \infty} e(t)=0$.

\section{Nonlinear Feedback Control of Chaotic Systems Based on Takagi-Sugeno Fuzzy Modelling}

The approach of differential flatness theory-based adaptivefuzzy control of chaotic systems is compared to control of such systems with the use of Takagi-Sugeno fuzzy modelling and the solution of Linear Matrix Inequalities (LMIs) [3]. It is shown that the differential flatness theory-based adaptivefuzzy control is more efficient because it is a control method based on exact global linearization of the chaotic oscillators' dynamics and thus avoids numerical approximators and truncation of specific terms in the linearized equivalent of the system. Besides, by succeeding the $H_{\infty}$ tracking performance criterion it provides improved robustness to modeling uncertainties and external disturbances.

After linearization round local operating points the initial nonlinear dynamical system of the form

$\dot{x}=f(x, u)$

is written in the form of Takagi-Sugeno fuzzy model, that is [45] 
Rule i : IF $x_{1}(t)$ is $M_{1}^{i} A N D x_{2}(t)$ is $M_{2}^{i} A N D \ldots$

$$
\begin{aligned}
& A N D x_{n}(t) \text { is } M_{n}^{i} \\
& T H E N \delta x(t)=A_{i} x(t)+B_{i} u(t) i=1,2, \cdots, r
\end{aligned}
$$

where $x_{j}$ is the $j$-th variable of the state vector, $M_{j}^{i}$ is the $i$-th fuzzy set into which the value range of the $j$-th input variable is partitioned, $x(t)=\left[x_{1}(t), \cdots, x_{n}(t)\right]^{T}$ in $R^{n}$ is the state vector, $u(t)=\left[u_{1}(t), \cdots, u_{m}(t)\right]^{T} \in R^{m}$ is the input vector, while it holds $A_{i} \in R^{n \times n}$ and $B_{i} \in R^{n \times m}$. It is noted that the model described in Eq. (56) includes also the autonomous system dynamics, that is when $u(t)=0$.

The output of the Takagi-Sugeno fuzzy model is

$\delta x(t)=\frac{\sum_{i=1}^{r} w_{i}\left[A_{i} x(t)+B_{i} u(t)\right]}{\sum_{i=1}^{r} w_{i}}$

where

$w_{i}=\prod_{k=1}^{r} \mu_{K}^{i}\left(x_{k}(t)\right)$

A condition that assures that the aforementioned system remains stable is stated as follows [45]:

Condition 1 The equilibrium of an autonomous TakagiSugeno fuzzy model is globally asymptotically stable if there exists a common symmetric positive definite matrix $P$, such that

$A_{i}^{T} P+P A_{i}<0 \forall i=1,2, \cdots, r$

Next, stability conditions are formulated for the case of a nonautonomous system. A feedback fuzzy controller is designed by using the antecedent part of the Takagi-Sugeno fuzzy model of the system. The fuzzy controller consists of the following rules

Rule j: IF $x_{1}(t)$ is $M_{1}^{j} A N D x_{2}(t)$ is $M_{2}^{j} A N D \ldots$

$$
\begin{aligned}
& A N D x_{n}(t) \text { is } M_{n}^{j} \\
& \quad T H E N u(t)=K_{j} x(t) j=1,2, \cdots, r
\end{aligned}
$$

The aggregate control signal that is applied to the nonlinear system becomes

$u(t)=\frac{\sum_{j=1}^{r} w_{j} K_{j} x(t)}{\sum_{j=1}^{r} w_{j}}$

By applying the above feedback control the output of the closed-loop system becomes

$\delta x(t)=\frac{\sum_{i=1}^{r} \sum_{j=1}^{r} w_{i} w_{j}\left(A_{i}+B_{i} K_{j}\right) x(t)}{\sum_{i=1}^{r} \sum_{j=1}^{r} w_{i} w_{j}}$
Stability conditions for the continuous-time Takagi-Sugeno fuzzy system under state feedback can be also formulated [45].

Condition 2 The equilibrium of the fuzzy system is globally asymptotically stable if there exists a common symmetric positive definite matrix $P$ such that

$$
\left(A_{i}+B_{i} K_{j}\right)^{T} P+P\left(A_{i}+B_{i} K_{j}\right)<0 \forall i, j=1,2, \cdots, r
$$

Finally, a less conservative condition about the stability of the continuous-time Takagi-Sugeno system is formulated as follows [45]

Condition 3 The equilibrium state for the continuous-time fuzzy system is globally asymptotically stable if there exists a common positive definite matrix $P$ such that

$$
\begin{aligned}
& \left(A_{i}+B_{i} K_{i}\right)^{T} P+P\left(A_{i}+B_{i} K_{i}\right)<0, i=1,2, \cdots, r \\
& \left(G_{i j}^{T} P+P G_{i j}\right)<0, i<j \leq r
\end{aligned}
$$

where

$G_{i j}=\frac{\left(A_{i}+B_{i} K_{j}\right)+\left(A_{j}+B_{j} K_{i}\right)}{2}, i, j \leq r$

\section{Simulation Tests}

\subsection{Flatness-Based Control}

The Lorenz chaotic system is considered as a case study. Among other application examples, the Lorenz system dynamics is met in the blood flow in the aorta in the case of an aneurysm. The control input is $\rho=u$. The phase diagrams of the Lorenz system when constant control input is applied are shown in Fig. 1. For the implementation of the differential flatness theory-based adaptive fuzzy control one has a fuzzy rule base of the form

$$
\begin{aligned}
& R^{l}: \text { IF } x_{1} \text { is } M_{1}^{l} \text { AND } x_{2} \text { is } M_{2}^{l} \text { AND } x_{3} \text { is } M_{3}^{l} \\
& \text { THEN } \hat{f}^{l} \text { is } b^{l}
\end{aligned}
$$

Regarding the implementation of the flatness-based adaptive fuzzy controller, the neurofuzzy approximators for functions $f(x, t)$ and $g(x, t)$ have now three inputs, i.e. $x_{1}, x_{2}$ and $x_{3}$. Taking that each fuzzy input variable consists of three fuzzy sets, there are now 27 fuzzy rules.

The performance of flatness-based control of the chaotic system was compared against feedback control that was based on describing the system's dynamics through local models and on solving LMIs. The nonlinear dynamics of the Lorenz system was described in Eq. (1) and the associated state vector was $\mathbf{x}=\left[x_{1}, x_{2}, x_{3}\right]=[x, y, z]$. The 

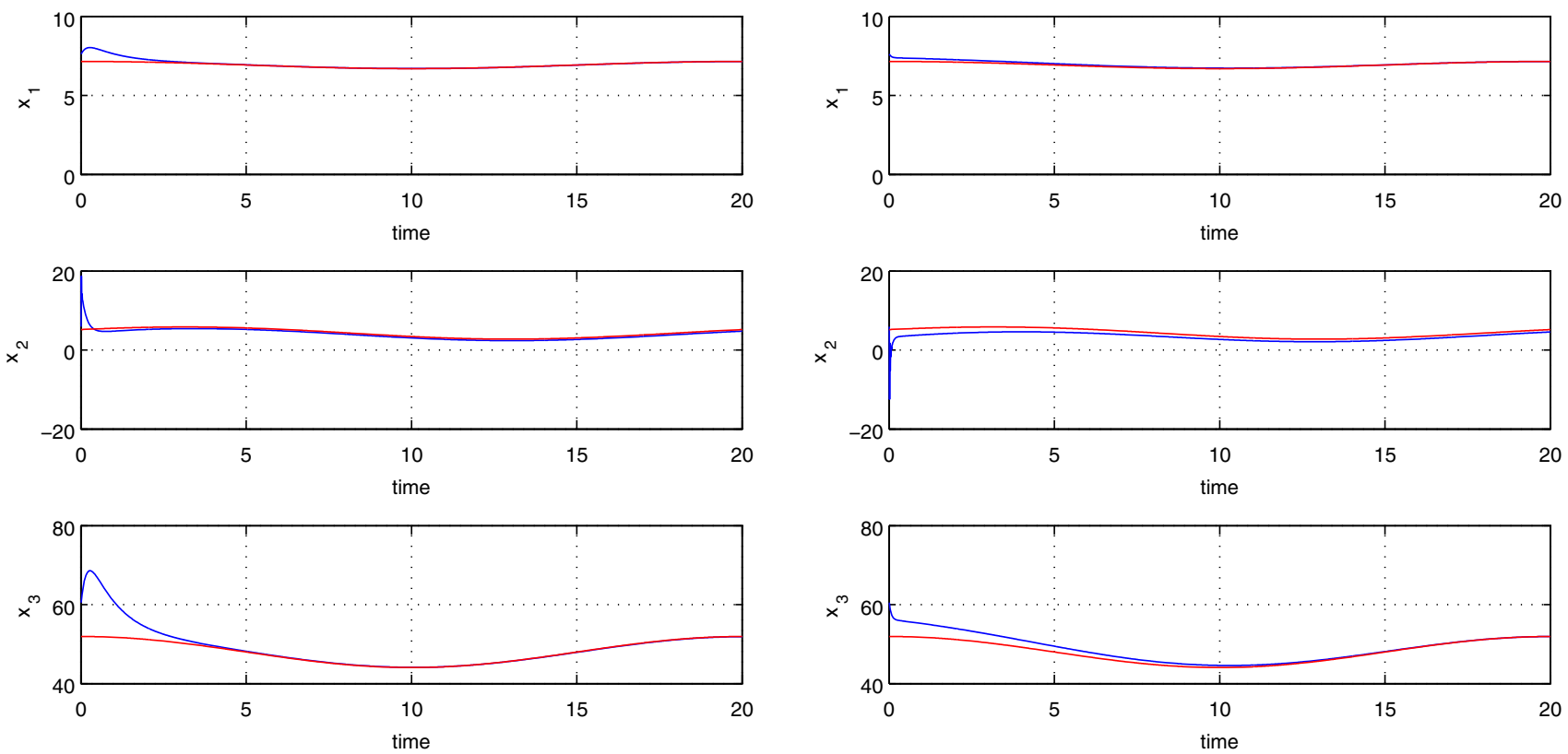

(a)

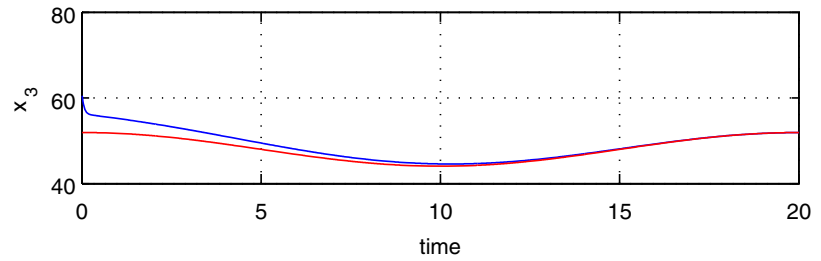

(b)

Fig. 4 Tracking of setpoint 1 from state variables $x_{i}, i=1, \cdots, 3$, a adaptive fuzzy control of the Lorenz system, $\mathbf{b}$ Takagi Sugeno model-based feedback control of the Lorenz system
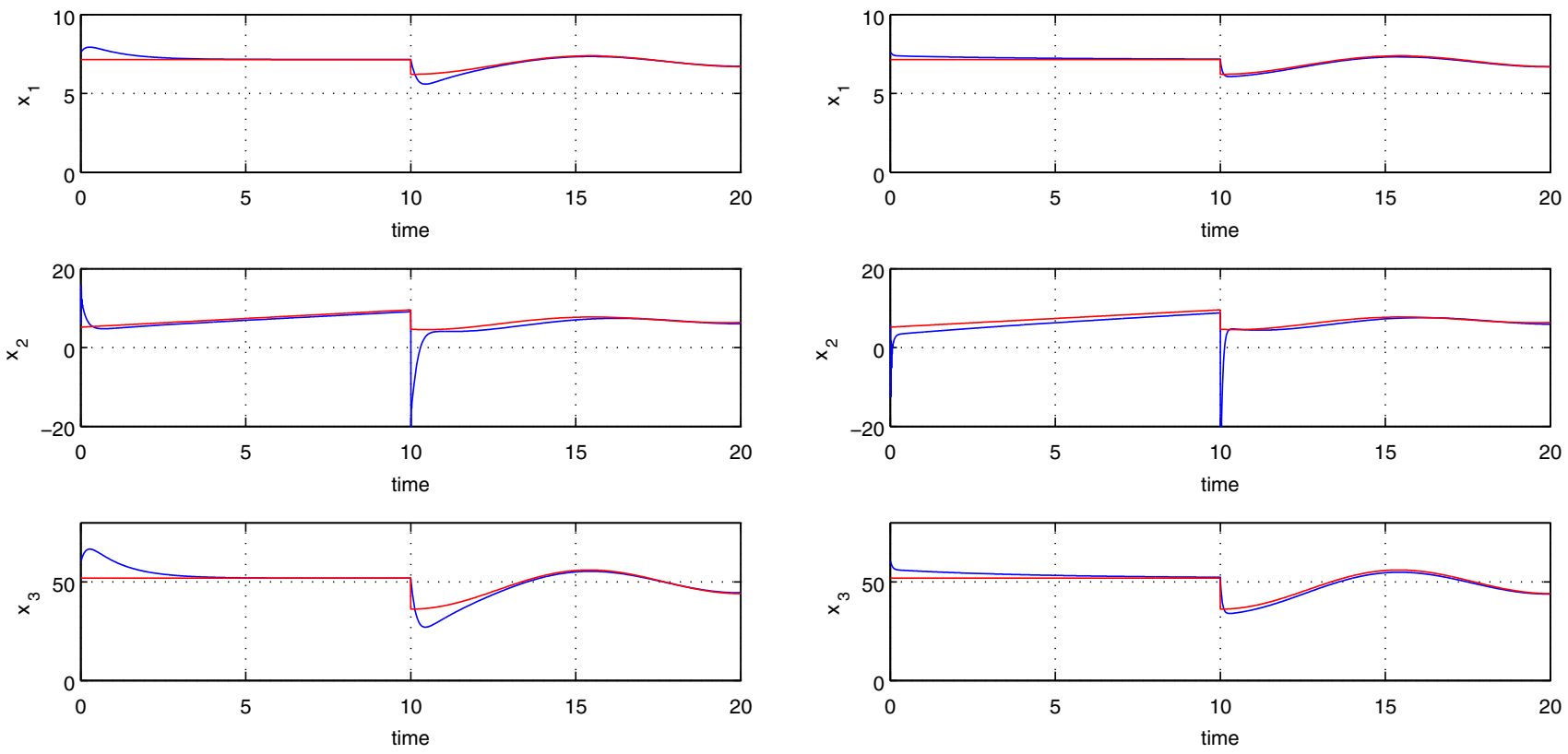

(a)

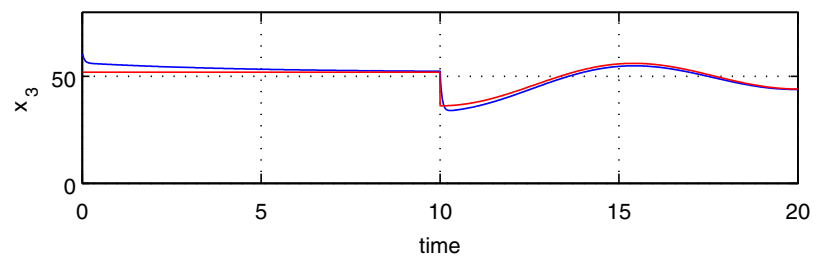

(b)

Fig. 5 Tracking of setpoint 2 from state variables $x_{i}, i=1, \cdots, 3$, a Adaptive fuzzy control of the Lorenz system, b Takagi Sugeno model-based feedback control of the Lorenz system

initial nonlinear model of the Lorenz system is in the form $\dot{x}=f(x)+g(x) u$. The nonlinear system was decomposed into four local models

$$
\dot{x}=A_{i} x+B_{i} u i=1, \cdots, 4
$$

$A_{i}=\left(\begin{array}{ccc}0 & \sigma & -\sigma \\ -x_{3} & -1 & -d_{i} \\ x_{2} & d_{i} & -\beta\end{array}\right) B_{i}=\left(\begin{array}{c}0 \\ d_{i} \\ 0\end{array}\right)$

where $d_{i}$ are specific points in the range of variation of the state variable $x_{1}=x$. Matrix $A_{i}$ if the Jacobian $J_{x} f(x)$ 

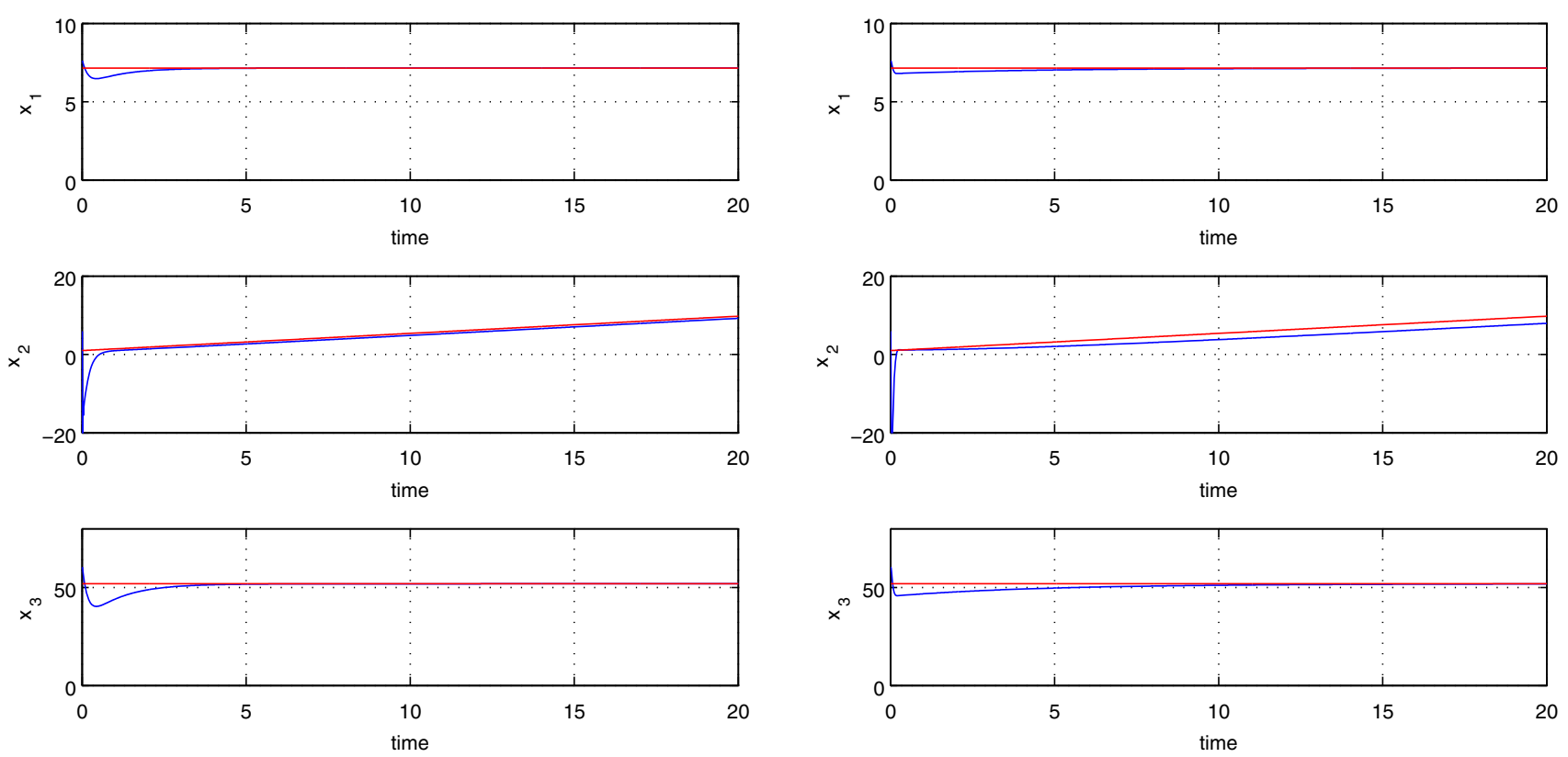

(a)

(b)

Fig. 6 Tracking of setpoint 3 from state variables $x_{i}, i=1, \cdots, 3$, a adaptive fuzzy control of the Lorenz system, b Takagi Sugeno model-based feedback control of the Lorenz system
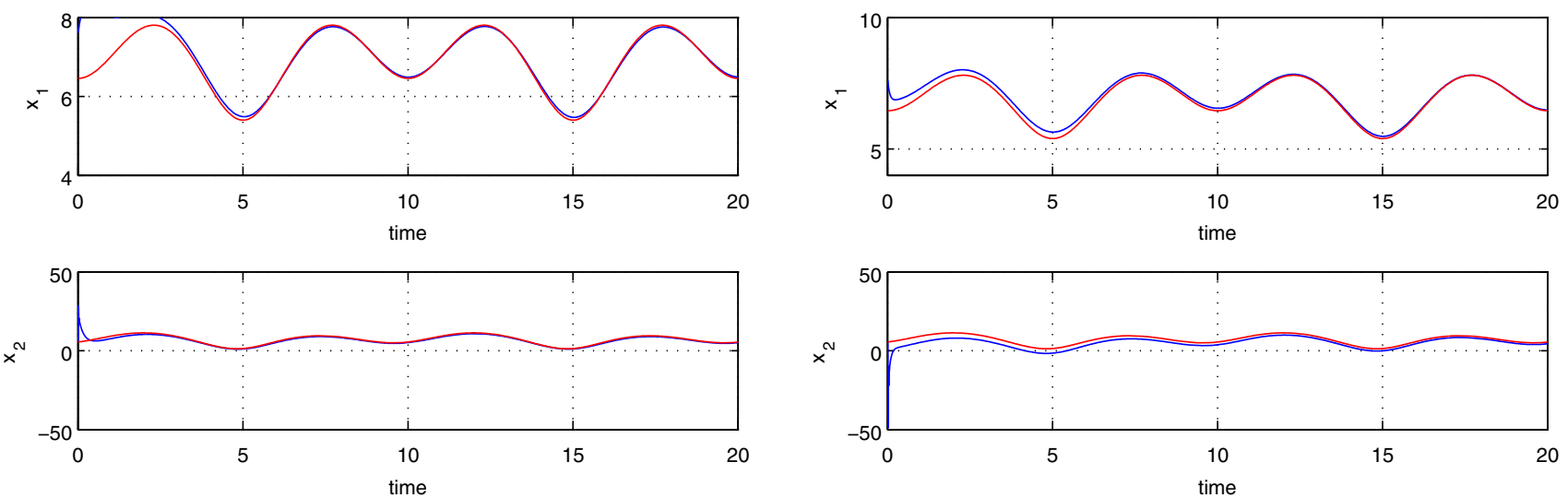

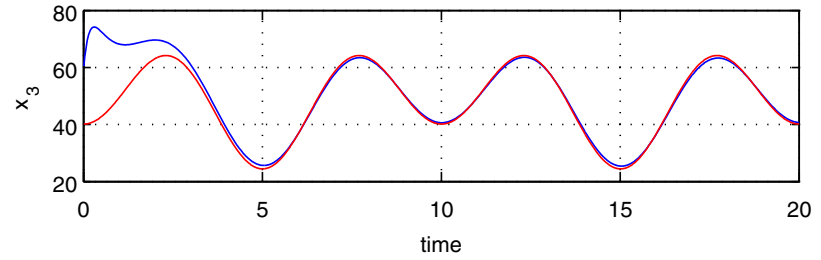

(a)

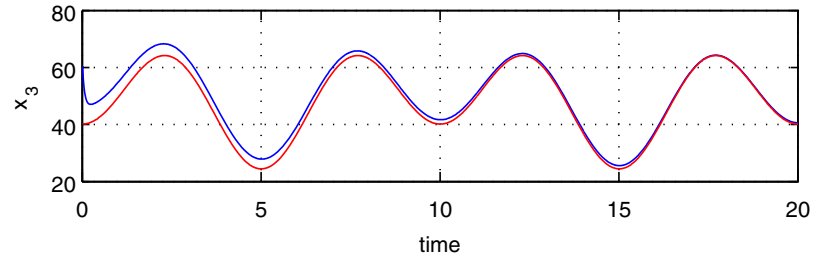

(b)

Fig. 7 Tracking of setpoint 4 from state variables $x_{i}, i=1, \cdots, 3$, a adaptive fuzzy control of the Lorenz system, b Takagi Sugeno model-based feedback control of the Lorenz system

computed at the points $d_{i} i=1, \cdots, 4$. Each linearization point $d_{i}$ is associated with a fuzzy set $M_{i}$ which is centered at $d_{i}$ and which has spread equal to $v_{i}$. The Takagi-Sugeno model of the Lorenz system takes the form

$\dot{x}=\frac{\sum_{i=1}^{4} \mu_{M_{i}}(x)\left[A_{i} x+B_{i} u\right]}{\sum_{i=1}^{4} \mu_{M_{i}}(x)}$
The results about the performance of the feedback control loop are depicted in Figs. 4, 5, 6, 7 and 8. It can be noticed, that differential flatness theory-based adaptive fuzzy control, succeeds fast and accurate tracking of the reference setpoints. The global linearization features of differential flatness theory enables transformation of the nonlinear system dynamics to an accurate equivalent linear model, thus also permitting 

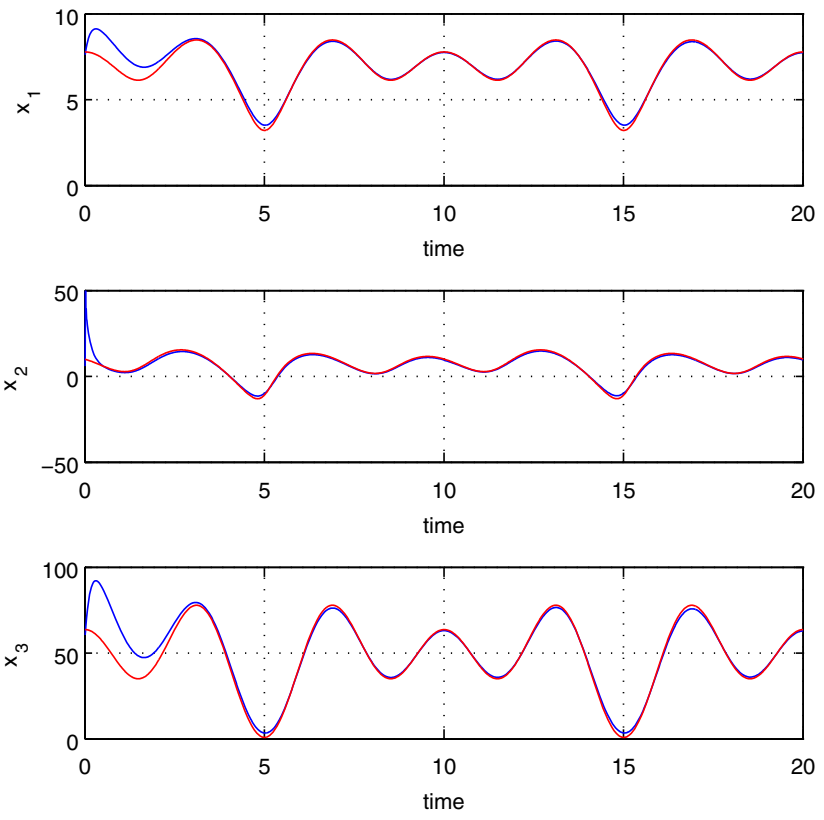

(a)
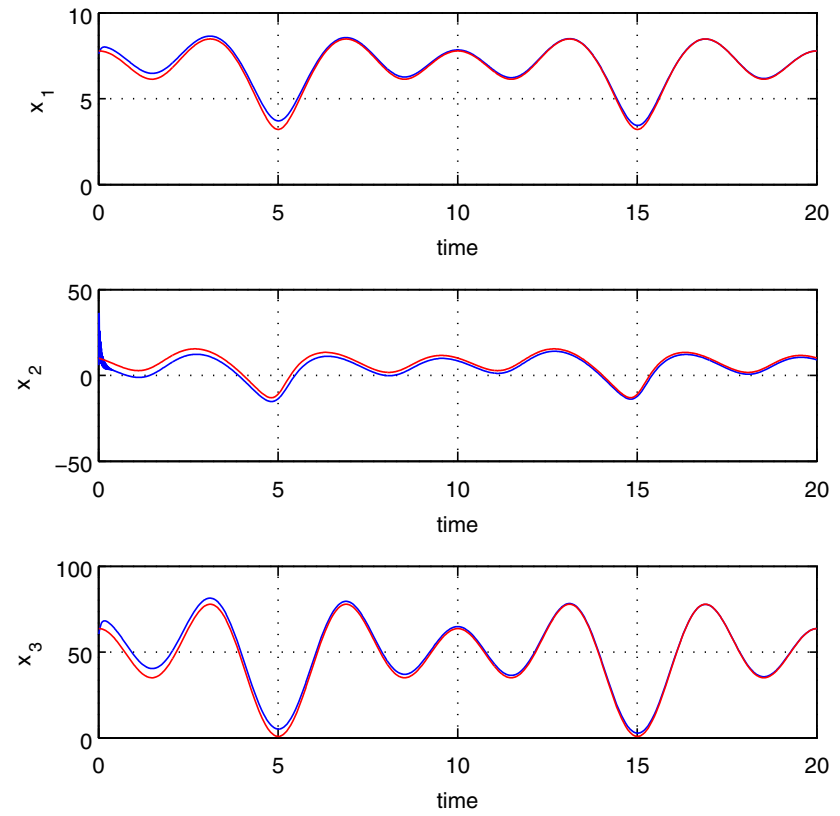

(b)

Fig. 8 Tracking of setpoint 5 from state variables $x_{i}, i=1, \cdots, 3$, a adaptive fuzzy control of the Lorenz system, $\mathbf{b}$ Takagi Sugeno model-based feedback control of the Lorenz system

the design of a more efficient state feedback controller. It is also pointed out that unlike Takagi-Sugeno model-based control, the adaptive fuzzy control based on differential flatness theory made no use of the mathematical model of the chaotic dynamical system (or of the associated linear local models). Although no knowledge about the model parameters was used, the differential flatness theory-based adaptive fuzzy control scheme succeeded better tracking performance.

\section{Conclusions}

In this paper, a new neurofuzzy adaptive control scheme for chaotic dynamical systems has been developed with the use of differential flatness theory. It has been shown that the dynamic model of the chaotic dynamical system (Lorenz oscillator) is a differentially flat one, which means that all state variables and the control inputs can be expressed as functions of a unique flat output variable and its derivatives. By proving that the model satisfies differential flatness properties it has been shown that it can be transformed to the linear canonical (Brunovsky) form. For the latter description the design of a state feedback controller becomes easier. Moreover, to cope with parametric uncertainties and external disturbances for the chaotic dynamical model, or even to treat the case that the system's model is completely unknown, the implementation of the adaptive fuzzy control scheme has been proposed .
After the description of the chaotic dynamical model in the canonical form, the associated transformed inputs where shown to contain unknown nonlinear functions which had to be identified online with the use of neurofuzzy approximators. It has been proven that a convergent adaptation law for the parameters of the neurofuzzy networks exists, as a result of the requirement to have a negative definite Lyapunov function for the closed control loop. Moreover, by using Lyapunov stability analysis it has been confirmed that the closed loop of the control system satisfies the $H_{\infty}$ tracking performance criterion. The latter assures improved robustness to external perturbations affecting the chaotic dynamical system. Finally, the efficiency of the proposed control scheme has been confirmed through simulation experiments.

\section{References}

1. Chang, Y.C.: A robust tracking control for chaotic Chua's circuits via fuzzy approach. IEEE Trans. Circuits Syst. Part I 48(7), 889895 (2001)

2. Kim, J.H., Hyun, C.H., Kim, E., Park, M.: Adaptive synchronization of chaotic systems based on T-S fuzzy model. IEEE Trans. Fuzzy Syst. 15(3), 359-369 (2007)

3. Wu, Z.G., Shi, P., Su, H., Chu, J.: Sampled-data fuzzy control of chaotic systems based on T-S Fuzzy model. IEEE Trans. Fuzzy Syst. 22(1), 153-163 (2014)

4. Sira-Ramirez, H., Luviano-Juarez, A., Cortes-Romero, J.: A disturbance rejection flatness-based linear output feedback control approach for tracking tasks of Chua's circuit, 50th IEEE Confer- 
ence on Decision and Control and European Control Conference (CDC-ECC), Orlando, Florida (2011)

5. Fradkov, A.I., Andrievsky, B., Andrievsky, A.: Practically stable observer-based synchronization of discrete-time chaotic systems over the limited-band communication channel, 3rd International Conference on Physics and Control PhysCon 2007, Potsdam, Sept 2007

6. Posznyak, A.S., Yu, W., Sanchez, E.N.: Identification and control of unknown chaotic systems via dynamic neural network. IEEE Trans. Circuits Syst. Part I 46(12), 1491-1495 (1999)

7. Khanesar, M.A., Teshnehlab, M., Kaynak, O.: Observer-based indirect model reference fuzzy control system with application to control of chaotic systems. J. Frankl. Inst. 350, 419-436 (2013)

8. Chen, B., Liu, X., Tong, S.: Adaptive fuzzy approach to control unified chaotic systems. Chaos Solitons Fractals 34, 1180-1187 (2007)

9. Khanesar, M.A., Teshnehlab, M., Kaynak, O.: Control and synchronization of chaotic systems using a novel indirect model reference fuzzy controller. Soft Comput. 16(7), 1253-1265 (2012)

10. Chen, D., Zhao, W., Sprott, J.C., Ma, X.: Application of TakagiSugeno fuzzy model to a class of chaotic synchronization and antisynchronization. Nonlinear Dyn. 73, 1495-1505 (2013)

11. Loria, A.: Control of the 4th order hyper-chaotic system with one input. Commun. Nonlinear Sci. Numer. Simul. 15(6), 1621-1630 (2010)

12. Zhang, X., Khadra, A., Li, D., Yang, D.: Impulsive stability of chaotic systems represented by Takagi-Sugeno model. Chaos Solitons Fractals 41(4), 1863-1869 (2009)

13. Wang, L.X.: A Course in Fuzzy Systems and Control. PrenticeHall, Englewood Cliffs (1998)

14. Crespo, L., Agrawal, S.: Differential flatness and cooperative tracking in the Lorenz System. In: Proceedings of the American Control Conference Denver, Colorado, USA, June 2003

15. Wai, R.J., Chang, J.M.: Implementation of robust wavelet-neuralnetwork sliding-mode control for induction servo motor drive. IEEE Trans. Ind. Electron. 50(6), 1317-1334 (2003)

16. Nounou, H.N., Rehman, H.: Application of adaptive fuzzy control to AC machines. Appl. Soft Comput. 7(3), 899-907 (2007)

17. Lin, Y.J., Wang, W.: Adaptive fuzzy control for a class of uncertain non-affine nonlinear systems. Inf. Sci. 177, 3901-3917 (2007)

18. Qi, R., Tao, G., Tan, C., Yao, X.: Adaptive control of discrete-time state-space TS fuzzy systems with general relative degree. Fuzzy Sets Syst. 217, 22-40 (2013)

19. Yang, Y., Zhou, C., Jia, X.: Robust adaptive fuzzy control and its application to ship roll stabilization. Inf. Sci. 142, 177-194 (2002)

20. Tong, S., Li, H.-X., Chen, G.: Adaptive fuzzy decentralized control for a class of large-scale nonlinear systems. IEEE Trans. Syst. Man Cybern. B 34(1), 770-775 (2004)

21. Fliess, M., Mounier, H.: Tracking control and $\pi$-freeness of infinite dimensional linear systems. In: Picci, G., Gilliam, D.S. (eds.) Dynamical Systems Control Coding and Computer Vision, vol. 258, pp. 41-68. Birkhaüser, Basel (1999)

22. Laroche, B., Martin, P., Petit, N.: Commande par platitude: Equations différentielles ordinaires et aux derivées partielles. Ecole Nationale Supérieure des Techniques Avancées, Paris (2007)

23. Lévine, J.: On necessary and sufficient conditions for differential flatness. Appl. Algebra Eng. Commun. Comput. 22, 47-90 (2011)

24. Martin, P., Rouchon, P.: Systèmes plats: planification et suivi des trajectoires. Journées X-UPS, École des Mines de Paris, Centre Automatique et Systèmes (1999)

25. Rudolph, J.: Flatness Based Control of Distributed Parameter Systems: Examples and Computer Exercises from Various Technological Domains. Shaker Verlag, Aachen (2003)

26. Sira-Ramirez, H., Agrawal, S.: Differentially Flat Systems. Marcel Dekker, New York (2004)
27. Villagra, J., d'Andrea-Novel, B., Mounier, H., Pengov, M.: Flatness-based vehicle steering control strategy with SDRE feedback gains tuned via a sensitivity approach. IEEE Trans. Control Syst. Technol. 15, 554-565 (2007)

28. Yue, H., Li, J.: Output-feedback adaptive fuzzy control for a class of nonlinear time-varying delay systems with unknown control directions. IET Control Theory Appl. 6, 1266-1280 (2012)

29. Cho, Y.W., Park, C.W., Kim, J.H., Park, M.: Indirect model reference adaptive fuzzy control of dynamic fuzzy-state space model. IET Proc. Control Theory Appl. 148(4), 273-282 (2005)

30. Rigatos, G., Al-Khazraji, A.: Flatness-Based Adaptive Fuzzy Control for MIMO Nonlinear Dynamical Systems. In: Nonlinear Estimation and Applications to Industrial Systems Control, Nova Publications (2011)

31. Rigatos, G.G.: Adaptive fuzzy control with output feedback for $H_{\infty}$ tracking of SISO nonlinear systems. Int. J. Neural Syst. 18(4), $1-16$ (2008)

32. Rigatos, G.G.: A Differential Flatness Theory Approach to Observer-Based Adaptive Fuzzy Control of MIMO Nonlinear Dynamical Systems, Nonlinear Dynamics. Springer, Berlin (2014)

33. Rigatos, G.G., Tzafestas, S.G.: Adaptive fuzzy control for the ship steering problem. J. Mechatron. 16(6), 479-489 (2006)

34. Rigatos, G.G.: Adaptive fuzzy control for non-linear dynamical systems based on differential flatness theory. IET Control Theory Appl. 6(17), 2644-2656 (2012)

35. Rigatos, G.G.: Adaptive fuzzy control of DC motors using state and output feedback. Electr. Power Syst. Res. 79(11), 1579-1592 (2009)

36. Yousef, H.A., Hamdy, M., Shafiq, M.: Flatness-based adaptive fuzzy output tracking excitation control for power system generators. J. Frankl. Ins. 350, 2334-2353 (2013)

37. Rigatos, G.G.: Modelling and Control for Intelligent Industrial Systems: Adaptive Algorithms in Robotics and Industrial Engineering. Springer, Berlin (2011)

38. Rigatos, G.G.: Advanced Models of Neural Networks: Nonlinear Dynamics and Stochasticity of Biological Neurons. Springer, Heidelberg (2013)

39. Rigatos, G.G.: Differential Flatness Approaches to Nonlinear Filtering and Control: Applications to Electromechanical Systems. Springer, New York (2015)

40. Rigatos, G., Zhang, Q.: Fuzzy model validation using the local statistical approach. Fuzzy Sets Syst. 60(7), 882-904 (2009)

41. Bassevile, M., Nikiforov, I.: Detection of Abrupt Changes: Theory and Applications. Prentice-Hall, Englewood Cliffs (1993)

42. Kurylowicz, A., Jaworska, I., Tzafestas, S.G.: Robust stabilizing control: an overview. In: Tzafestas, S.G. (ed.) Applied Control: Current Trends and Modern Methodologies, pp. 289-324. Marcel Dekker, New York (1993)

43. Lublin, L., Athans, M.: An experimental comparison of and designs for interferometer testbed. In: Francis, B., Tannenbaum, A. (eds.) Lectures Notes in Control and Information Sciences: Feedback Control, Nonlinear Systems and Complexity, pp. 150-172. Springer, New York (1995)

44. Doyle, J.C., Glover, K., Khargonekar, P.P., Francis, B.A.: Statespace solutions to standard $H_{2}$ and $H_{\infty}$ control problems. IEEE Trans. Autom. Control 34, 831-847 (1989)

45. Farinwata, S., Filev, D., Langari, R.: Fuzzy Control: Synthesis and Analysis. Wiley, Chichester (2000) 